\title{
Relazione tra disfunzione erettile e ischemia miocardica silente in pazienti diabetici: studio angiografico coronarico mediante tomografia assiale computerizzata multistrato
}

\author{
Antonio Aversa ${ }^{1}$
}

Pubblicato online: 20 dicembre 2016

(c) Springer International Publishing AG 2016

Commento a:

Relation between erectile dysfunction and silent myocardial ischemia in diabetic patients: a multidetector computed tomographic coronary angiographic study.

A. Abdelhamed, S. Hisasue, E.A. Nada, A.M. Kassem, M. Abdel-Kareem, S. Horie.

Sex Med (2016) 4:e127-e134

È oramai ben noto che la disfunzione erettile (DE) può precedere la malattia coronarica. Inoltre, l'ischemia cardiaca silente $(\mathrm{SMI})$ è più frequente nei pazienti diabetici, ed è un forte fattore predittivo di eventi cardiaci e morte [1]. L'obiettivo del presente studio è stato quello di valutare la presenza di SMI in pazienti affetti da diabete e disfunzione erettile utilizzando l'angiografia coronarica mediante tomografia assiale computerizzata multistrato (MDCT-CA). Lo studio ha valutato 20 pazienti affetti da diabete e DE senza alcuna storia di sintomi o segni cardiaci. La funzione erettile è stata valutata mediante i questionari Sexual Health Inventory for Men score (SHIM) e Erection Hardness Score (EHS), e dalla variazione della circonferenza del pene mediante studio delle erezioni notturne mediante rigidometro.

Nei 20 pazienti studiati (età media 61 anni), la MDCT$\mathrm{CA}$ ha mostrato una stenosi dell'arteria coronarica in 13 soggetti $(65 \%)$. Tali soggetti erano altresì affetti da malattia monovasale nel $30 \%$ dei casi $(n=6)$, da malattia bivasale nel $10 \%$ dei casi $(n=2)$ e da malattia trivasale nel $25 \%$ dei casi $(n=5)$. Inoltre, è stato riscontrato che circa il $50 \%$ dei pazienti presentava una restrizione del lume dell'arteria

$\triangle$ A. Aversa

aversa@unicz.it

1 Dipartimento di Medicina Sperimentale e Clinica, Università di Catanzaro Magna Graecia, Catanzaro, Italia coronaria discendente sinistra di circa il $50 \%$; questa arteria risultava il vaso maggiormente colpito (55\%). Il 15\% (3 su 20) dei pazienti presentava una stenosi maggiore del $90 \%$, e due di essi sono stati sottoposti ad angioplastica coronarica immediata con posizionamento di stent per prevenire l'infarto miocardico. Gli autori hanno riscontrato che la massima stenosi dell' arteria coronaria correla positivamente con l'età e negativamente con il grado di rigidità peniena (all'EHS), dimostrando inoltre che l'effetto età-correlato era il solo fattore predittivo positivo di SMI. Questo studio pilota presenta notevoli limitazioni. Il numero di pazienti è limitato sia per numero sia per campione (pazienti affetti da DE) e non sono stati valutati i risultati rispetto a un gruppo di controllo senza diabete. Non è stato calcolato l'indice di calcificazione delle coronarie e, infine, nonostante i livelli di testosterone siano stati valutati, non è stata effettuata nessuna correlazione tra questi e lo score di calcificazione coronarico, dato esistente in letteratura [2]. In conclusione, la MDCT-CA si è dimostrata uno strumento utile per l'identificazione della SMI in pazienti diabetici con disfunzione erettile, in particolare in quelli di età avanzata e/o con grave ED. Se tali dati verranno confermati in studi controllati su larga scala, potrebbero rappresentare un mezzo efficace per migliorare la durata della vita a numerosi pazienti diabetici di età avanzata e con lunga durata di malattia, anche in assenza di sintomi cardiaci.

\section{Bibliografia}

1. Gazzaruso C1, Solerte SB, De Amici E et al (2006) Association of the metabolic syndrome and insulin resistance with silent myocardial ischemia in patients with type 2 diabetes mellitus. Am J Cardiol 97:236-239

2. Rosano GM, Sheiban I, Massaro R et al (2007) Low testosterone levels are associated with coronary artery disease in male patients with angina. Int J Impot Res 19:176-182 